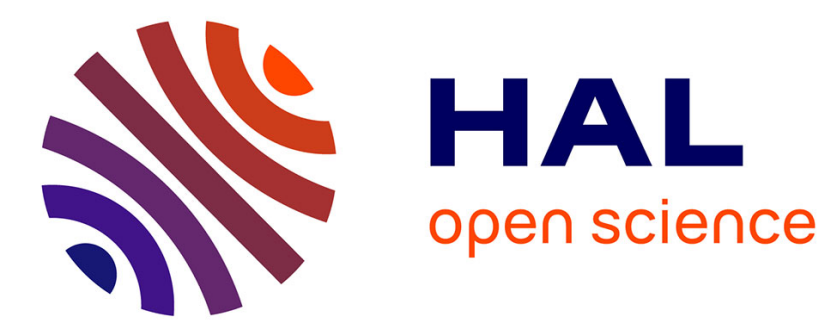

\title{
VEA: A Virtual Environment for Animal experimentation
}

Lahcen Oubahssi, Oussema Mahdi

\section{To cite this version:}

Lahcen Oubahssi, Oussema Mahdi. VEA: A Virtual Environment for Animal experimentation. The 21th IEEE International Conference on Advanced Learning Technologies (ICALT 2021), Jul 2021, Online, France. pp.422-424, 10.1109/ICALT52272.2021.00134 . hal-03381590

\section{HAL Id: hal-03381590 https://hal.science/hal-03381590}

Submitted on 17 Oct 2021

HAL is a multi-disciplinary open access archive for the deposit and dissemination of scientific research documents, whether they are published or not. The documents may come from teaching and research institutions in France or abroad, or from public or private research centers.
L'archive ouverte pluridisciplinaire HAL, est destinée au dépôt et à la diffusion de documents scientifiques de niveau recherche, publiés ou non, émanant des établissements d'enseignement et de recherche français ou étrangers, des laboratoires publics ou privés. 


\title{
VEA: A Virtual Environment for Animal experimentation
}

\author{
Lahcen Oubahssi \& Oussema Mahdi \\ Le Mans Université, LIUM, EA 4023, Laboratoire d'Informatique de l'Université du Mans, \\ Avenue Messiaen, 72085 LE MANS CEDEX 9, France. \\ lahcen.oubahssi@,univ-lemans.fr, oussema.mahdi@,univ-lemans.fr
}

\begin{abstract}
This research work is part of the VEA (Virtual Environment for Animal experimentation) project. The aim of this project was to develop an alternative method based on virtual reality for learning the correct gestures in animal experimentation while respecting the rules of ethics (the Rule of 3Rs). Our goal was to facilitate this learning via digital means, and to provide trainers with an educational toolkit that allowed them to recreate virtual reality scenarios and to assess each learner's progress. In this paper, we are particularly interested in the design and operationalisation of learning situations in virtual environments, and propose solutions in the form of virtual action models that allow the user to learn the correct gestures.
\end{abstract}

Keywords: VEA, Animal Experimentation, Pedagogical simulation, Virtual Reality, Virtual Learning Environments.

\section{INTRODUCTION}

The potential benefits of VR in terms of conducting educational activities have been explored over the past decade. Learning environments that use VR techniques are known as VRLE (virtual reality environments for human learning). These allow teachers to exploit the potential of VR and to create original and dynamic teaching situations that are free from the constraints that may exist in real training scenarios, such as risk or uncertainty. In our research, we are particularly interested in the educational design of VRoriented activities. To anticipate the unfolding of this type of learning activity, the teacher must pay particular attention to the pedagogical treatment, which is based on an explanation of the pedagogical scenario [1]. When designing the scenario, the teacher must also anticipate the interaction between the different components of the learning scenario, such as the activities, resources, tools and roles involved [1], to ensure that it creates the desired mental processes in the learners. In this paper, we present research carried out as part of the VEA (Virtual Environment for Animal experimentation) project, conducted in collaboration with the Biological Engineering department at the Laval Institute of Technology, France. The aim of this project is to propose an alternative method based on VR to allow the user to learn the correct gestures while respecting the rules of ethics (the Rule of 3Rs)[2][3]. Our goal is to facilitate this learning through digital means, and to provide trainers with an educational toolkit that allows them to recreate VR scenarios and to assess the learner's progress. In this paper, we are particularly interested in the design and operationalisation of learning situations in virtual environments, and propose solutions in the form of virtual action models that allow the user to learn correct gestures. This article is divided into six sections: in Section 2, we discuss the relevant scientific background; Section 3 presents the educational context to the project; Section 4 describes the virtual action model; Section 5 describes the main functionalities of the developed prototype, which includes our model of action to allow teachers to design and adapt their pedagogical situations; and Section 6 presents the conclusion.

\section{SCIENTIFIC BACKGROUND}

We are interested in the interaction (virtual action) between humans and the virtual world (virtual objects). An educational activity is a succession of actions that a learner must accomplish in a virtual environment. Thus, to describe virtual educational activities, it is sufficient to describe the actions corresponding to each activity. In addition, the fact that a user can act in the virtual environment constitutes the key element that justifies the use of VR in learning situations, from our point of view. According to Patel [4], the aim of interaction and immersion is to promote the learning of gestures and behaviours through situational awareness and the transfer of skills from virtual to real. These concepts are rarely perfectly feasible in a given application, and this aim is mostly an objective to be achieved as far as possible. However, they must be realised, even modestly, in a system based on VR techniques. The identification of user actions is complex in VR, because it is necessary both to formalise the way in which interactions are supported and to specify the VR devices that will allow these actions to be realised. According to [5], there are three levels of immersion and interaction: sensorimotor immersion and interaction $\left(\mathrm{I}^{2}\right)$, cognitive $\mathrm{I}^{2}$, and functional $\mathrm{I}^{2}$. Depending on the environment used, the user (learner) must perform actions that can always be broken down into a few basic behaviours called virtual behavioural primitives (VBPs). VBPs form the objectives at the functional level of $\mathrm{I}^{2}$ [6]. The concept of a VBP has been described in detail in [7]. For optimal use, we refine the VBPs into sub-categories, each containing specific VR actions. We also associate some of the most frequently recommended VR devices with each category [8]. The VRLE designer can therefore choose the VR actions to be realised by the learner, the information that is presented, and the devices that are provided to the learners in order to accomplish the actions and to facilitate their learning as much as possible. The user performs VBPs using virtual devices, such as a VR controller and any other element that can create sensory stimuli and allow the user to interact with 
a virtual environment in the same way as in a real environment.

\section{EDUCATIONAL CONTEXT}

In France, the BBA (Biological and Biochemical Analysis) option of the National Educational Program of the DUT Biological Engineering is composed of different modules relating to animal physiology, pharmacology and toxicology, and includes training in animal experimentation that is distributed across two years of study. One of the main objectives of these modules is to train students in the approaches used to experiment on animals. Animal experimentation [2] consists of using animals as a surrogate or model to better understand the physiology of an organism and its responses to various factors (e.g. food, environment, pathogens) or substances, in order to test, verify or evaluate their performance, efficacy, safety or toxicity. Research on animal models is regulated at the legislative and regulatory level. European and French legislation have been the subject of regular revision due to increasing concerns over animal welfare [3]. As part of this project, we developed a virtual environment for animal experimentation (a small mammal model). The main objective of this research is to offer students and teachers in the Biological Engineering department of the Laval University Institute of Technology, France, an alternative VR method of learning the correct gestures while respecting the rules of ethics (the 3Rs rule) [3]. We focused on the example of an educational situation consisting of placing a catheter in a canal, vein or artery of an anesthetised animal. To achieve this, the learner must carry out a set of activities, for example: (1) anaesthesia of the animal via intraperitoneal injection; (2) placing the animal in the supine position; (3) fixing the animal; (4) incising (cutting) the skin from the shoulder girdle to the base of the chin; (5) introducing a tracheal catheter; etc. The format of this paper (three pages) does not allow us to describe the design and development of all these activities in detail. Before presenting the functionalities of the VAE environment, we first describe our action model, and illustrate it with the example of a virtual cutting action.

\section{VAE: WHAT VIRTUAL ACTION MODEL?}

To describe an educational situation, a teacher identifies the educational activities involved and the corresponding educational objectives. He or she then defines the actions to be realised by the learners. We use the term action to describe an interaction with the virtual environment and the related cause-and-effect relationships. Within a virtual environment, an action establishes the necessary link between the use of the virtual environment and the learning activity. The proposed action model can serve as a reference for the learner's conception of an educational activity, and for the scripted development of events that may occur within the virtual environment. In our model (Figure 1), each action has a virtual object, which may be pedagogical or raw. In its turn, each object has a specific role involving the object, on which it acts, the change in its position due to the action, the object through which the learner can generate the action, etc. These roles have semantic labels and are called thematic roles. As illustrated in Figure 1, the VR action entity VRAction is the central entity of the action model. It is characterised by a well-defined pedagogical objective, a description, and instructions that must be followed in order to perform the action properly. We note that some actions must be performed in a predefined order. Actions can be divided into four categories of VBPs identified in Section 2. In order to describe these actions in more detail, we have used reference shapes called checkpoints. These may be spherical or cubic, depending on the needs of the teacher. To represent them, we use interfaces that define a noninstantiable class structure.

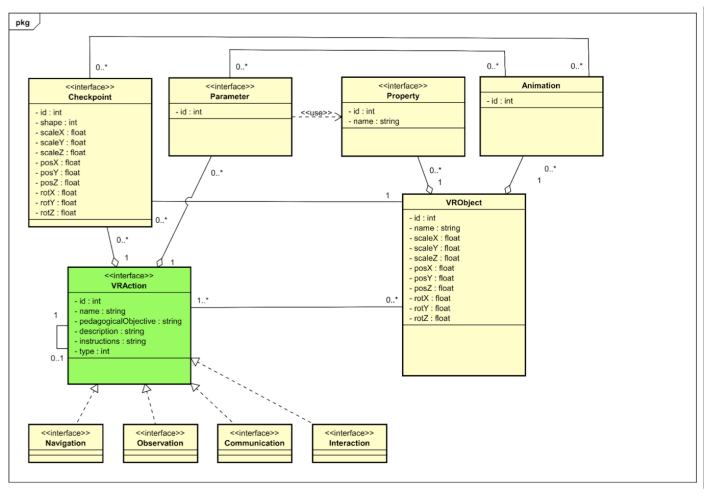

Figure 1. The VR action model

\section{VAE: EXAMPLE OF AN EDUCATIONAL ACTIVITY}

In this example of an educational situation, the objective is to place a catheter in a canal, vein or artery in an anesthetised animal. The learner is led to carry out the cut action to open the skin of the animal (a rat) from the shoulder girdle to the chin base. This action requires two virtual objects, the scissors instrument, used to perform the cutting action, and the rat object, on which the action is performed.

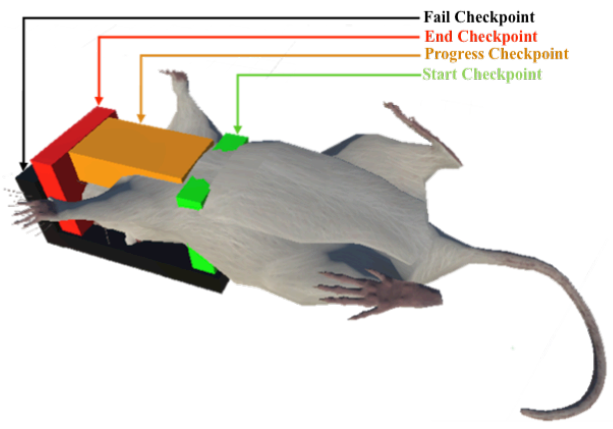

Figure 2. Checkpoints used when cutting the skin of an animal

In order to describe this action more precisely, we use four checkpoints (Figure 2): StartCheckpoint, which designates the start of the action; ProgressCheckpoint, which measures the progress of the action; EndCheckpoint, which marks the end of the action; and FailCheckpoint, which reflects an unsuccessful action by the learner. In this example, the type of checkpoint is cubic. In the case where the learner has successfully completed the cut action, an 
animation is triggered to show that this action has been successfully completed. The teacher defines one or more Fail checkpoints to specify zones in which actions were not correctly carried out. Figure 2 shows the four checkpoints used for the cut action.

\section{VAE: FunCTIONALITIES}

The design, development and evaluation of the VAE application were realised using an agile iterative process in several phases. The environment allows learners to train in a virtual world on various educational activities as part of their practical work (both within the laboratory and in a real context), and also allows the teacher to configure and adapt certain activities according to the students' needs.

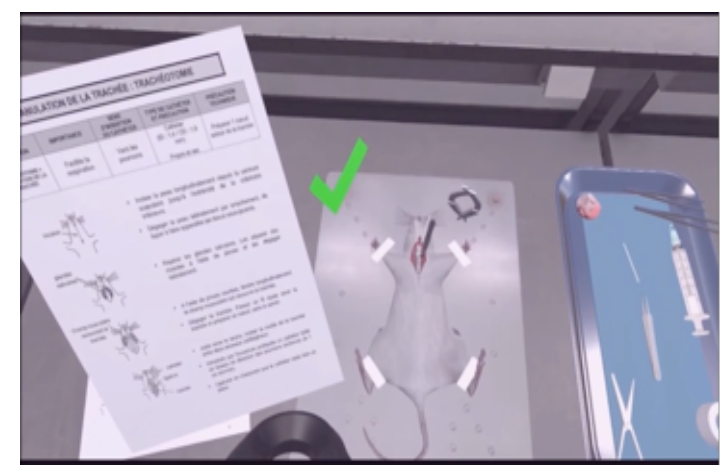

Figure 3. The main Interface of VEA

The main functionalities of VAE are: (1) Connecting: to connect, the user (teacher or learner) must use a VR headset with a VR controller, which allows them to affect the environment and its objects; (2) Moving within the virtual laboratory: once immersed in the VR environment, the user can move freely, and can also use the teleport function to go from one place to another; (3) Visualising and manipulating virtual objects: with the two VR controllers, the learner can manipulate the different laboratory objects, and in particular the tools that are required to realise the practical work on animal experimentation; (4) Using the practical worksheet: to understand the different activities that need to be realised, the learner can consult the steps listed in the practical worksheet; (5) Time spent: this functionality allows the learner to be aware of the time spent on each activity. The teacher can associate a particular duration with each educational activity or virtual action; (6) Access to educational resources: the teacher can provide the learner with educational capsules (in video format). This functionality can aid the learner in realising their practical work; (7) Validation of realised actions: this functionality indicates (in real time) whether or not the action realised by the learner is correct, based on the concept of checkpoints, as described in previous sections. Figure 3 illustrates the main interface of VAE. An link is provided to an illustration of the overall VAE functionalities. ${ }^{1}$

${ }^{1}$ http://perso.univ-lemans.fr/ loubah/videos/VideoRatPresentation.mp4

\section{CONCLUSION}

VR offers new experiences to users via increasingly efficient methods of interaction and immersion. These are of great interest in the domain of education. This work was based on a co-design process in which several teachers from the Biological Engineering department teaching team participated in the design of different VAE models through an iterative, user-centred approach. The elements developed in this project provide an experimental framework for our research work. Our objective is to study issues related to the design and operationalisation of pedagogical situations in VRLEs, and to propose solutions that can assist teachers in designing, reusing and deploying their pedagogical scenarios in a VRLE [8]. Our aim is to offer technical and methodological solutions that are reusable and adaptable, i.e. that can be applied in numerous environments, regardless of the field or the type of task to be completed. The process of designing and producing a VRLE must consider the pedagogical requirements of teachers in order to fulfil their needs [8]. We carried out experiments throughout the development process to verify that the functionalities and interaction methods were well adapted to students, and that they allowed for easy operation and assessment of one or more skills. Proposals for improvement were integrated into the final version of the VAE tool, and the teaching team is very satisfied with the results. In the context of the COVID19 pandemic, we were not able to test the application with first year Biological Engineering students, and this experiment has been postponed to a later date.

\section{ACKNOWLEDGEMENTS}

The current work is supported by the Laval University Institute of Technology, France. The authors want to thank all the persons who have contributed to this project.

\section{REFERENCES}

[1] J-P. Pernin, and A. Lejeune, "Models for the re-use of learning scenarios", in Imagining the future for ICT and Education, IFIP conference Proceedings, Ålesund, Norway, June 2006.

[2] R. Kolar, “Animal experimentation”. SCI ENG ETHICS 12, 111-122 (2006). https://doi.org/10.1007/s11948-006-0011-1

[3] https://ec.europa.eu/environment/chemicals/lab_animals/3r/alternativ e en.htm

[4] H. Patel, O. Stefani, S. Sharples, H. Hoffmann, I. Karaseitanidis, and A. Amditis, "Human centred design of 3-D interaction devices to control virtual environments" Int. J. Human-Computer Studies 64 (2006) 207-220. https://doi.org/10.1016/j.ijhcs.2005.08.010

[5] P. Fuchs, F. Nashashibi, and D. Lourdeaux, "Three levels of Immersion and Interaction in a Approach of the VR Design", International Journal of Design and Innovation Research, IJDIR, 2002, 2 (1), pp. 30-42.

[6] S. Richir, P. Fuchs, D. Lourdeaux, D. Millet, and C. Buche, "How to design compelling Virtual Reality or Augmented Reality experience?", International Journal of Virtual Reality, IPI Press, 2015, 15(1), pp.35-47. hal-01273284.

[7] M. Mine, "Virtual Environment Interaction Techniques", UNC Chapel Hill. Computer Science Technical Report, TR95-018. 1995

[8] O. Mahdi O, L. Oubahssi, C. Piau-Toffolon and S. Iksal, "Towards an editor for VR-oriented educational scenarios", in the 14th European Conference on Technology Enhanced Learning, Delft, the Netherlands, 16-19 September 2019. 